\title{
Fine-tuned iron availability is essential to achieve optimal adipocyte differentiation and mitochondrial biogenesis
}

\author{
José María Moreno-Navarrete • Francisco Ortega • \\ María Moreno • Wifredo Ricart • José Manuel Fernández-Real
}

Received: 19 March 2014 / Accepted: 27 May 2014 /Published online: 29 June 2014

(C) Springer-Verlag Berlin Heidelberg 2014

\begin{abstract}
Aims/hypothesis Adipose tissue from obese and insulinresistant individuals showed altered expression of several iron-related genes in a recent study, suggesting that iron might have an important role in adipogenesis. To investigate this possible role, we aimed to characterise the effects of iron on adipocyte differentiation.

Methods Intracellular iron deficiency was achieved using two independent approaches: deferoxamine administration (20 and $100 \mu \mathrm{mol} / \mathrm{l}$ ) and transferrin knockdown (TF KD). The effects of added $\mathrm{FeSO}_{4}$, holo-transferrin and palmitate were studied during human and 3T3-L1 adipocyte differentiation. Finally, the relationship between iron-related and mitochondrialrelated genes was investigated in human adipose tissue.

Results Most adipose tissue iron-related genes were predominantly expressed in adipocytes compared with stromal vascular cells. Of note, transferrin gene and protein expression increased significantly during adipocyte differentiation. Both
\end{abstract}

Electronic supplementary material The online version of this article (doi:10.1007/s00125-014-3298-5) contains peer-reviewed but unedited supplementary material, which is available to authorised users.

J. M. Moreno-Navarrete · F. Ortega $\cdot$ M. Moreno $\cdot$ W. Ricart $\cdot$

J. M. Fernández-Real

Department of Diabetes, Endocrinology and Nutrition, Institut d'Investigació Biomèdica de Girona (IdIBGi), Hospital of Girona

'Dr Josep Trueta', Girona, Spain

J. M. Moreno-Navarrete $\cdot$ F. Ortega $\cdot$ M. Moreno $\cdot$ W. Ricart $~$

J. M. Fernández-Real

CIBEROBN (CB06/03/010), Centro de Investigación Biomédica en

Red de Fisiopatología de la Obesidad y Nutrición (CIBEROBN)

URL: www.ciberobn.es/

\section{J. M. Fernández-Real ( $₫)$}

Section of Diabetes, Endocrinology and Nutrition, Hospital of

Girona 'Dr Josep Trueta', Carretera de França s/n,

17007 Girona, Spain

e-mail: jmfreal@idibgi.org deferoxamine and TF KD severely blunted adipocyte differentiation in parallel with increased inflammatory mRNAs. These effects were reversed in a dose-dependent manner after iron supplementation. Palmitate administration also led to a state of functional intracellular iron deficiency, with decreased Tf gene expression and iron uptake during adipocyte differentiation, which was reversed with transferrin co-treatment. On the other hand, iron in excess impaired differentiation, but this antiadipogenic effect was less pronounced than under iron chelation. Of interest, expression of several genes involved in mitochondrial biogenesis occurred in parallel with expression of iron-related genes both during adipogenesis and in human adipose tissue.

Conclusions/interpretation Precise and fine-tuned iron availability is essential to achieve optimal adipocyte differentiation, possibly modulating adipocyte mitochondrial biogenesis.

Keywords 3T3-L1 $\cdot$ Adipocytes $\cdot$ Adipogenesis $\cdot$ Adipose tissue $\cdot$ Ferritin $\cdot$ Iron $\cdot$ Knockdown $\cdot$ Mitochondrial biogenesis $\cdot$ Transferrin

$\begin{array}{ll}\text { Abbreviations } \\ \text { FTL } & \text { Light ferritin } \\ \text { KD } & \text { Knockdown } \\ \text { SAT } & \text { Subcutaneous adipose tissue } \\ \text { shRNA } & \text { Short hairpin RNA } \\ \text { SVF } & \text { Stromal vascular cell fraction } \\ \text { TF KD } & \text { Transferrin knockdown } \\ \text { VAT } & \text { Visceral adipose tissue }\end{array}$

Introduction

Iron seems to play a direct and causal role in the pathophysiology of type 2 diabetes mediated by both beta cell failure and 
insulin resistance [1-6]. Iron in excess impacts on systemic metabolism across the entire spectrum of iron stores. The association of iron overload with increased risk of type 2 diabetes mellitus has been extensively demonstrated in recent systematic reviews and meta-analysis [5-8]. Circulating markers of iron overload are also positively associated with visceral and subcutaneous fat depots [9] and with adipocyte insulin resistance [10]. A previous study using the 3T3-L1 mouse cell line suggested the importance of iron-related genes in adipocyte physiology [11]. Interestingly, recent studies in mice revealed that an iron-enriched diet induced iron accumulation and insulin resistance in visceral adipose tissue (VAT) [12] and that adipocyte iron overload reduced adiponectin biosynthesis in parallel with induction of insulin resistance [13]. Another recent study in mice showed impaired macrophage iron handling in the setting of obesity-associated adipose tissue dysfunction, which resulted in adipocyte iron uptake and overload [14]. Despite these associations, little attention has been paid to the molecular mechanisms regulating iron homeostasis in human adipose tissue.

In a recent study, we found that obese and insulin-resistant individuals showed increased light ferritin (FTL) and SLC40A1, an iron exporter, in their subcutaneous adipose tissue (SAT) and VAT, in parallel with decreased transferrin $(T F)$ gene expression. These results suggest that iron accumulates in adipose tissue in humans with increased body fatness and impaired insulin action [15], as previously demonstrated in mice [12-14].

At the cellular level, iron has been recently identified as a key regulator of mitochondrial biogenesis [16]. In fact, intracellular iron is used in the build-up of Fe-S clusters, which are necessary for the regulation of mitochondrial oxidative processes [17].

Interestingly, the mitochondrial biogenesis programme is a key metabolic process in adipocyte differentiation [18] even though the role of mitochondria in regulating adipocyte function has received relatively little attention. Reduced mitochondrial mass and function has been found in adipose tissue of $o b / o b$ and $d b / d b$ mice and in adipose tissue from obese individuals with type 2 diabetes in association with increased activity of several inflammatory pathways [18-24]. Given previous findings in whole adipose tissue, we hypothesised that iron might have an important role in adipogenesis, and we decided to explore in greater depth which cells are responsible for the altered expression of genes involved in iron metabolism and to investigate the precise role of iron in adipocyte differentiation. For this reason, the effects of iron depletion and excess during silencing of target genes during adipocyte differentiation were evaluated. Furthermore, to elucidate the effect of metabolic perturbation on iron metabolism during adipocyte differentiation, the effects of saturated fatty acids (palmitate) on these genes were also tested. Since iron is essential for mitochondrial biogenesis [16], and optimal mitochondrial biogenesis and function is a key component in adipocyte differentiation [18], we also analysed genes related to mitochondrial biogenesis in these experiments.

\section{Methods}

Recruitment of participants for adipose tissue samples A group of 43 paired VAT and SAT samples from obese nondiabetic participants recruited by the Endocrinology Service of the Hospital of Girona 'Dr Josep Trueta' (Girona, Spain) were analysed. All participants were white and reported that their body weight had been stable for at least 3 months before the study. All gave written informed consent, validated and approved by the ethics committee of the Hospital of Girona 'Dr Josep Trueta', after the purpose of the study had been explained to them. Samples were obtained from SAT and VAT depots during elective surgical procedures (cholecystectomy, surgery for abdominal hernia, and gastric bypass surgery) and transported immediately to the laboratory $(5-10 \mathrm{~min})$. Tissue samples were handled under strictly aseptic conditions. They were washed in PBS, cut up with forceps and scalpel into small pieces $(100 \mathrm{mg})$, and immediately flash-frozen in liquid nitrogen before being stored at $-80^{\circ} \mathrm{C}$.

Adipocytes and stromal vascular cell fraction (SVF) were isolated from eight SAT and eight VAT non-frozen samples as described previously [25] and in electronic supplementary material (ESM) Methods.

Differentiation of human pre-adipocytes Isolated human subcutaneous pre-adipocytes (Zen-Bio, Research Triangle Park, NC, USA) were cultured and differentiated as described previously [25] and in ESM Methods.

Differentiation of mouse cell line, 3T3-L1 The embryonic fibroblast mouse cell line, 3T3-L1 (American Type Culture Collection), was cultured and differentiated as described previously [25] and in ESM Methods. Palmitate (100 and $250 \mu \mathrm{mol} / \mathrm{l})$, palmitate $(100$ and $250 \mu \mathrm{mol} / \mathrm{l})+$ $\mathrm{FeSO}_{4}$ (3 and $30 \mu \mathrm{g} / \mathrm{ml}$ ), and palmitate $(100$ and $250 \mu \mathrm{mol} / \mathrm{l})+$ holo-transferrin $(0.1$ and $1 \mu \mathrm{g} / \mathrm{ml})$ were administered throughout the whole 3T3-L1 adipocyte differentiation process. Palmitate doses were selected according to the physiological range of palmitate concentration and other in vitro studies [26, 27].

Iron addition and chelation experiment Human and 3T3-L1 pre-adipocytes and adipocytes were incubated with fresh medium (control), fresh medium containing $\mathrm{FeSO}_{4}$ (3 and $30 \mu \mathrm{g} / \mathrm{ml})$, deferoxamine $(20$ and $100 \mu \mathrm{mol} / \mathrm{l})$, and the following combinations: $\mathrm{FeSO}_{4}(3 \mu \mathrm{g} / \mathrm{ml})+$ deferoxamine $(20 \mu \mathrm{mol} / \mathrm{l}), \mathrm{FeSO}_{4}(30 \mu \mathrm{g} / \mathrm{ml})+$ deferoxamine $(20 \mu \mathrm{mol} / \mathrm{l})$ 
and $\mathrm{FeSO}_{4}(30 \mu \mathrm{g} / \mathrm{ml})+$ deferoxamine $(100 \mu \mathrm{mol} / \mathrm{l})$. These experiments were performed during human and 3T3-L1 adipocyte differentiation (14 days for human pre-adipocytes and 7 days for the 3T3-L1 cell line, and adding the appropriate amount in each medium change). At the end of the experiment, the supernatant fractions were centrifuged at $400 \mathrm{~g}$ for $5 \mathrm{~min}$, the cells were harvested, and pellets and supernatant fractions were stored at $-80^{\circ} \mathrm{C}$ for RNA analysis. All these treatments were performed in four independent replicates.

Short hairpin RNA-mediated knockdown of TF and FTL Permanent silencing (knockdown; KD) was performed using $T f$ - and $F t l$-targeted and control short hairpin (sh)RNA lentiviral particles (sc-37177-V, sc-40578-V and sc-108080; Santa Cruz Biotechnology, Santa Cruz, CA, USA) and following the manufacturer's instructions. Positive 3T3-L1 preadipocytes harbouring the shRNA cassette for $T f$ or $F t l$ were selected with puromycin $(3 \mu \mathrm{g} / \mathrm{ml}) 60 \mathrm{~h}$ after infection.

Oil red staining Intracellular lipid accumulation was measured by Oil red staining as described in ESM Methods.

Iron measurement Iron concentration in conditioned medium and levels of intracellular iron (from cell lysates) were measured using a specific colorimetric commercial assay (Iron-Ferrozine, ref. 11509; BioSystems, Barcelona, Spain) according to the manufacturer's instructions.

$R N A$ expression RNA purification, gene expression procedures and analyses, and primer/probe sets used were as previously reported [25] and briefly described in ESM Methods.

Statistical analysis Statistical analyses were performed using SPSS 12.0 software. Descriptive results of continuous variables are expressed as mean \pm SEM. Paired Student's $t$ test and non-parametric tests (Mann-Whitney $U$ test) were used to evaluate in vitro experiments. For human cross-sectional studies, descriptive results of continuous variables are expressed as mean $\pm \mathrm{SD}$, and the relation between variables was analysed by simple correlation (using Spearman's test). Levels of statistical significance were set at $p<0.05$.

\section{Results}

Iron-related gene expression in human adipose tissue fractions and during adipocyte differentiation TF, FTL and FTHI were predominantly expressed in adipocytes compared with the SVF (Fig. 1). TFRC and SLC4OA1 were mainly expressed in SVF cells (Fig. 1). TF gene expression and transferrin protein secretion increased significantly during adipocyte differentiation, attaining the highest levels at the end of the
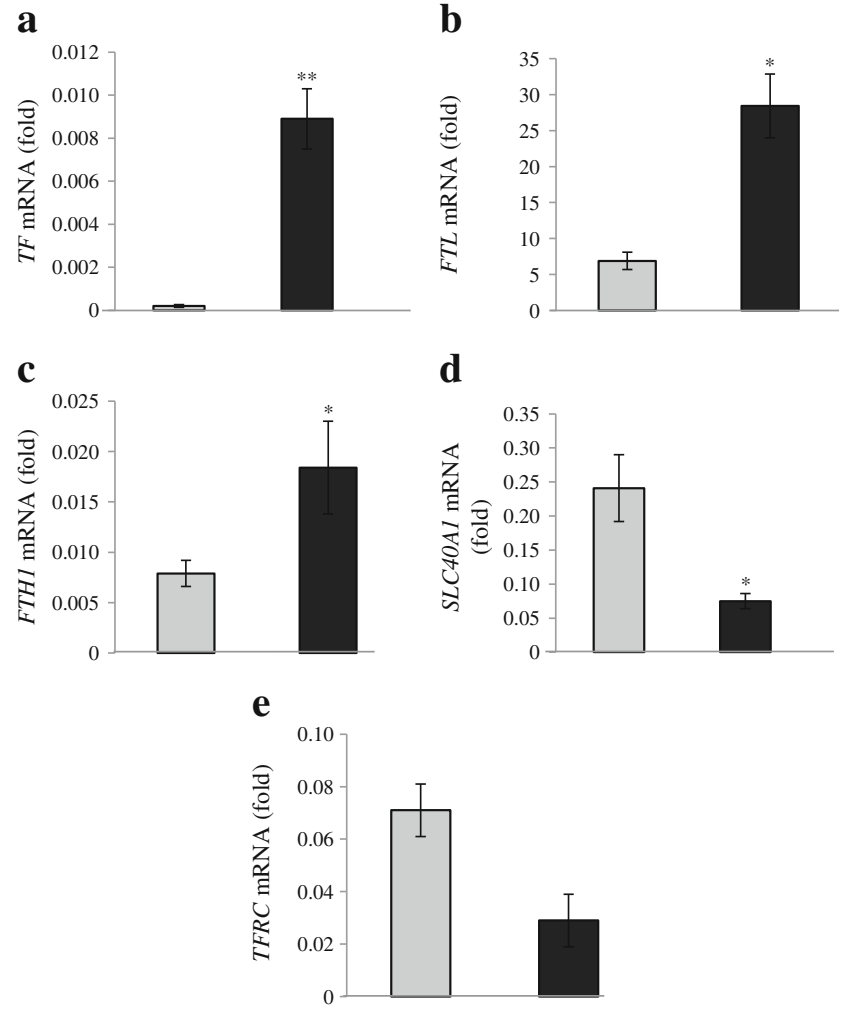

Fig. 1 TF (a), FTL (b), FTH1 (c), SLC40A1 (d) and TFRC (e) gene expression in adipose tissue fractions (SVF [grey bars] vs mature adipocytes [black bars]). ${ }^{*} p<0.05,{ }^{* *} p<0.01$ compared with SVF. These data are expressed as mean $\pm \mathrm{SEM}$. Relative gene expression is expressed as fold change (fold)

process in parallel with the gene expression pattern of other adipogenic genes (ADIPOQ, FASN) (Fig. 2). TF expression and the rate of transferrin biosynthesis in subcutaneous adipocytes was significantly higher than in visceral adipocytes $\left(4.11 \pm 0.18\right.$ vs $0.19 \pm 0.03$ pg $10^{3}$ cells $^{-1} \mathrm{~h}^{-1}$; $p<0.0001)$. FTL, FTH1 and SLC40A1 increased significantly in the first 2 days of adipocyte differentiation, and decreased thereafter (Fig. 2). No significant changes were found in $T F R C$ gene expression during adipocyte differentiation.

These results were replicated in 3T3-L1 cells and are described in ESM Results and ESM Fig. 1.

Effects of TF and FTL KD on adipogenesis Tf and Ftl gene expression were decreased significantly in shRNA Tf- $(95 \%$, $p<0.0001)$ and shRNA Ftl- $(75 \%, p<0.0001)$ silenced 3T3L1 cells (Fig. 3). Iron concentration in the medium decreased significantly during adipocyte differentiation, this decrease being significantly attenuated in TF KD cells (Fig. 3). Furthermore, increased Tfrc and decreased $F$ th 1 gene expression was found in TF KD cells compared with control cells (ESM Fig. 2), suggesting a possible intracellular iron deficiency. During adipocyte differentiation, TF KD led to a significant decrease in the intracellular lipid accumulation at day 7 
$\mathbf{a}$

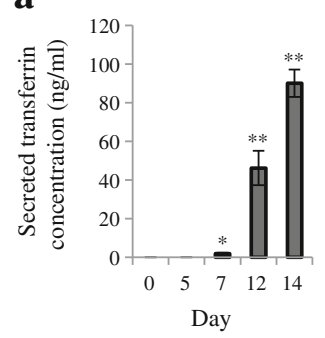

e

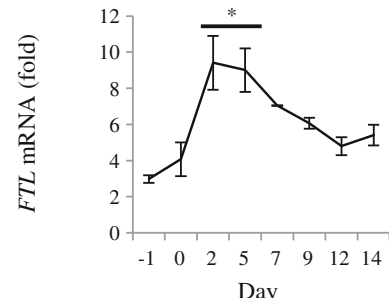

b

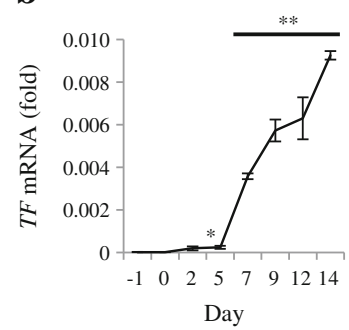

$\mathbf{f}$

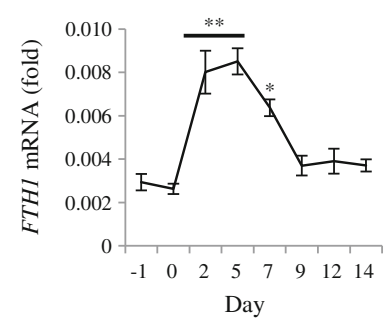

c

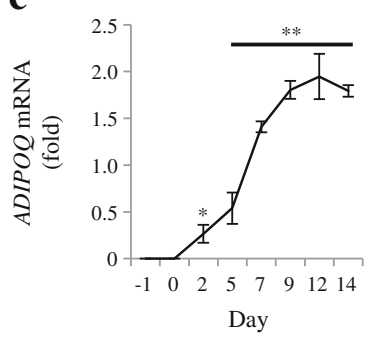

$\mathbf{g}$

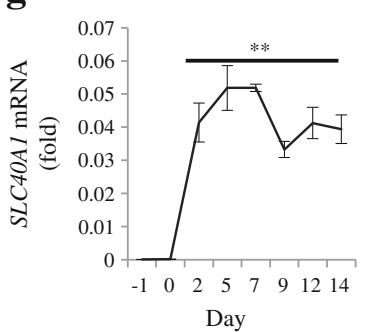

d

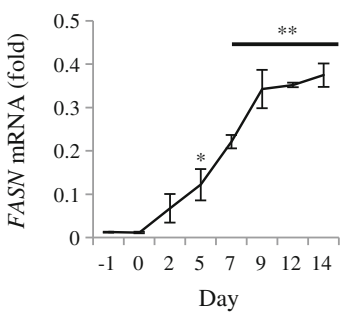

$\mathbf{h}$

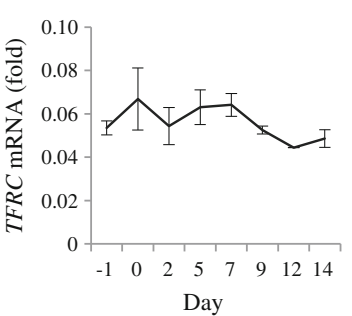

Fig. 2 Secreted transferrin concentration in the medium (a) and $T F(\mathbf{b})$, $A D I P O Q$ (c), FASN (d), FTL (e), FTHI (f), SLC4OAl (g) and TFRC (h) gene expression in a time course experiment during human subcutaneous

(Fig. 3) and in adipogenic mRNAs (such as Pparg, Adipoq, Glut4, Fabp4 and Cebpa) (Fig. 3) while increasing inflammatory (Mcpl and IlO) and Lep gene expression (Fig. 3). In contrast, no significant effects were found in FTL KD cells with regard to adipogenic gene expression (Fig. 3). Similarly to $T f$ depletion, Ftl depletion also led to increased Il6 at day 2 and Mcpl at day 0 (Fig. 3). Interestingly, expression of Ppargcla and Tfam was increased during adipocyte differentiation, and they were significantly decreased in both TF and FTL KD cells (Fig. 3 and ESM Fig. 2).

Effects of iron during adipogenesis Several doses and combinations of $\mathrm{FeSO}_{4}(3$ and $30 \mu \mathrm{g} / \mathrm{ml})$ and the iron chelator, deferoxamine (20 and $100 \mu \mathrm{mol} / \mathrm{l}$ ), were used in human preadipocytes and in the 3T3-L1 cell line during adipocyte differentiation.

As expected, $\mathrm{FeSO}_{4}(3$ and $30 \mu \mathrm{g} / \mathrm{ml})$ administration led to increased intracellular iron levels, whereas deferoxamine exerted opposite effects (Fig. 4). Deferoxamine-induced intracellular iron deficiency was reversed with $\mathrm{FeSO}_{4}$ coadministration (Fig. 4). Interestingly, iron excess and iron chelation led to either a slight decrease in adipocyte differentiation or pronounced antiadipogenic effects, respectively, decreasing intracellular lipid accumulation (Fig. 4) in parallel with decreased adipogenic gene expression and increased inflammatory mRNAs (Fig. 5 and ESM Fig. 3). Iron replacement reversed the antiadipogenic effects of deferoxamine (Figs 4 and 5 and ESM Fig. 3). As expected, added iron and iron chelation led to reciprocal changes in Tfrc (Fig. 5). Iron chelation also resulted in decreased Slc40al and increased Fth 1 and $F t l$ in these antiadipogenic conditions (Fig. 5). Tf followed a similar gene expression pattern to other adipogenic

adipocyte differentiation. ${ }^{*} p<0.05,{ }^{*} p<0.01$ compared with day 0 . These data are expressed as mean \pm SEM. Relative gene expression is expressed as fold change (fold)

genes with iron chelation (Fig. 5), while $T f$ gene expression decreased with excess iron.

In human pre-adipocytes, similar effects of iron in excess or chelation were found (Fig. 6 and ESM Fig. 4). Similarly to adipogenic gene expression, both iron addition and chelation led to decreased mitochondrial gene expression (PPARGC1A, PPARGC1B and MT-CO3) in human adipocytes, this reduction being more pronounced under iron chelation (Fig. 6 and ESM Fig. 4). The connection between iron metabolism and mitochondrial biogenesis is the Fe-S clusters, which are essential cofactors for the activity of key enzymes of the mitochondrial respiratory chain and of the tricarboxylic acid cycle. Iron-sulphur cluster assembly 2 (ISCA2) is involved in the maturation of mitochondrial iron-sulphur proteins. Strikingly, exogenous iron addition led to decreased ISCA2 gene expression, whereas iron chelation caused opposite effects (ESM Fig. 4).

Iron chelation, in parallel with the negative effects on adipogenesis and mitochondrial biogenesis, also led to increased expression of both endoplasmic reticulum stress (HSPA5) and inflammatory markers (IL8, ILO) (Fig. 6 and ESM Fig. 4).

In 3T3-L1 pre-adipocytes, similar effects of iron in excess or chelation were found on Ppargcla and Tfam and inflammatory (Il6 and Mcpl) gene expression (Fig. 5 and ESM Fig. 3).

Effects of iron in 3T3-L1 TF and FTL KD cells Next, we tested the effects of iron excess and iron chelation during adipogenesis of KD cells. These data are described in ESM Results and ESM Figs 5 and 6. 
a

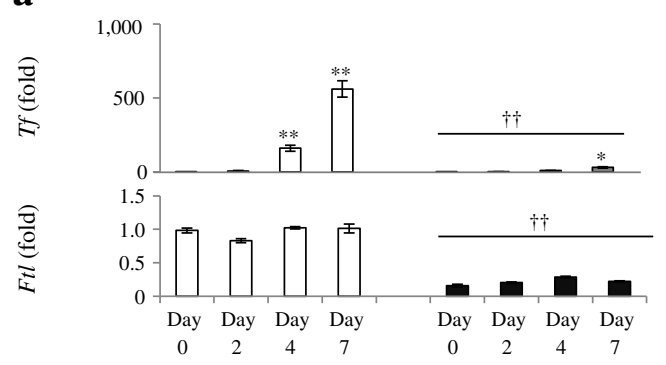

b

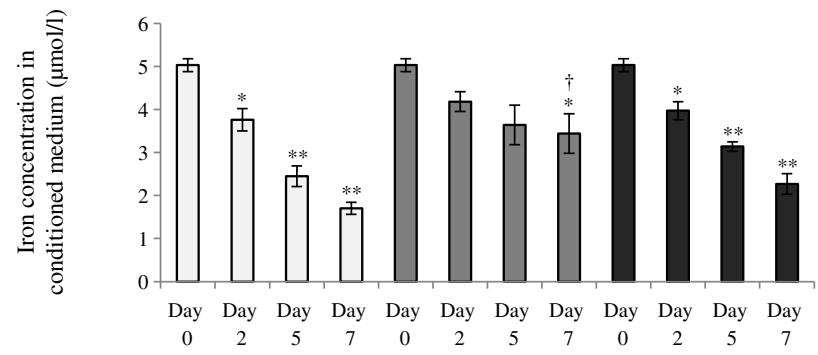

c
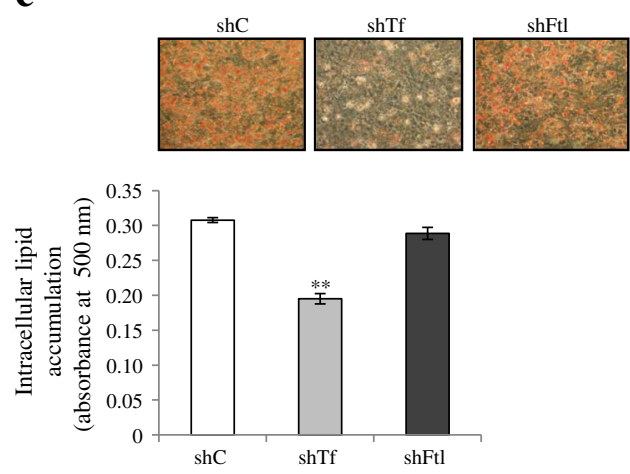

Fig. 3 Effects of TF and FTL KD on $T f$ and $F t l$ expression (a), iron concentration in the conditioned medium (an indirect measure of iron uptake) (b), intracellular lipid accumulation measured using Oil red staining (c), adipogenic, Ppargcla, inflammatory and Lep (d) gene expression during 3T3-L1 adipocyte differentiation in a time course

Palmitate effect on iron metabolism-related genes during adipocyte differentiation Palmitate (100 and $250 \mu \mathrm{mol} / \mathrm{l})$ administration significantly decreased transferrin gene expression, similarly to adipogenic genes (Fig. 7), and led to reduced iron uptake, intracellular iron levels being significantly reduced (Fig. 7) and iron concentration increased in the medium (ESM Fig. 7). The maximum dose of palmitate $(250 \mu \mathrm{mol} / \mathrm{l})$ produced a significant increase in $T f r c$ and $I l 6$ and a significant decrease in Slc40al gene expression. No significant effects were found on Fthl and Ftl (Fig. 7).

Interestingly, holo-transferrin $(0.1$ and $1 \mu \mathrm{g} / \mathrm{ml})$ but not $\mathrm{FeSO}_{4}(3$ and $30 \mu \mathrm{g} / \mathrm{ml}$ ) co-administration reversed the negative effects of palmitate on adipocyte differentiation (Fig. 7).

Genes related to iron metabolism and mitochondrial biogenesis in adipose tissue Given the marked effects of d
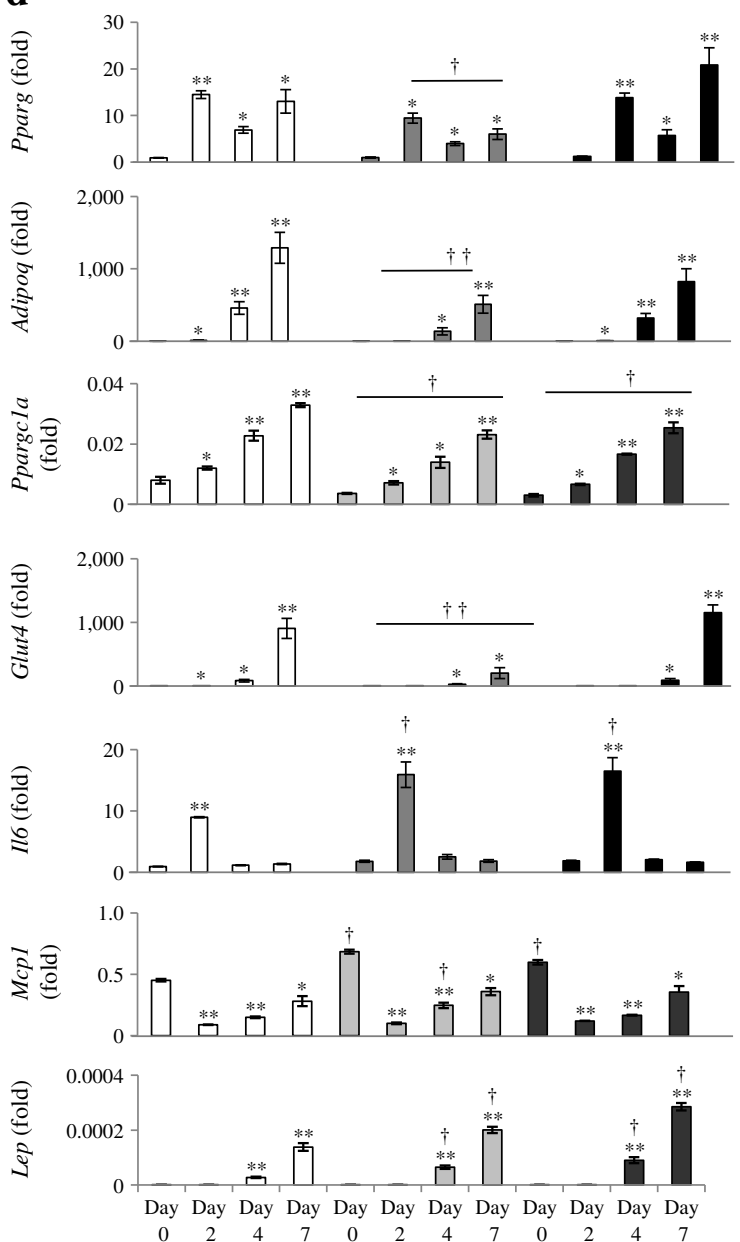

experiment. shC (white bars), shTf (grey bars) and shFtl (black bars) 3T3-L1 cells. ${ }^{*} p<0.05, * * p<0.01$ compared with day $0 .{ }^{\dagger} p<0.05$, ${ }^{\dagger \dagger} p<0.01$ compared with shC. These data are expressed as mean $\pm \mathrm{SEM}$. Relative gene expression is expressed as fold change (fold)

iron on adipocyte differentiation and mitochondrial biogenesis, we decided to explore these relationships in human adipose tissue $(n=43)$. Anthropometric and clinical variables of this cohort are provided in Table 1. These data are described in ESM Results.

\section{Discussion}

In a recent study, we found that several gene markers for intracellular iron accumulation (FTL, FTH and SLC40A1) were increased in adipose tissue from individuals with obesity and insulin resistance, while gene expression of transferrin (a mediator of iron uptake) was significantly reduced [15]. To try to understand these findings, we designed the present study 
b

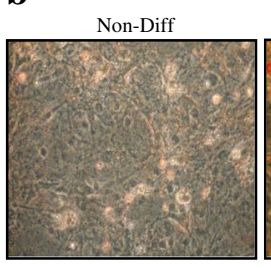

Fe30

a
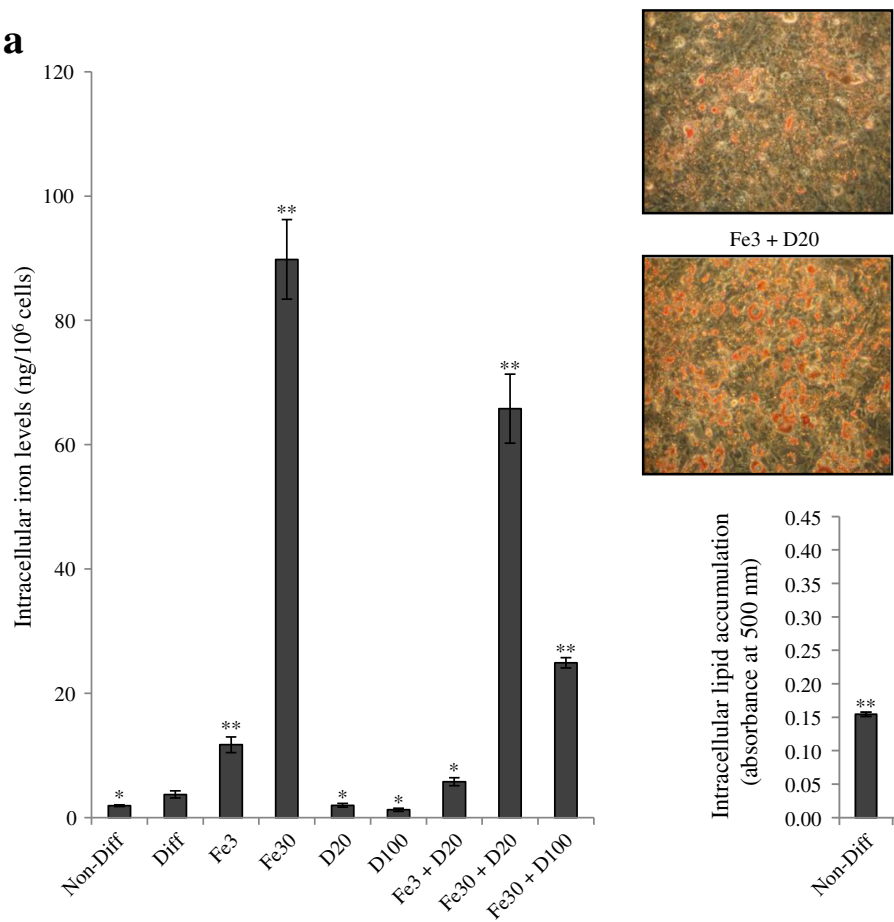

$\mathrm{Fe} 3+\mathrm{D} 20$
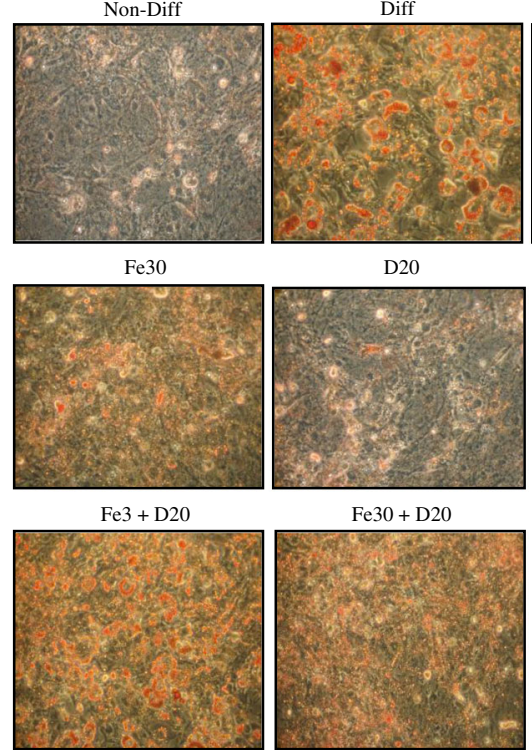

D20

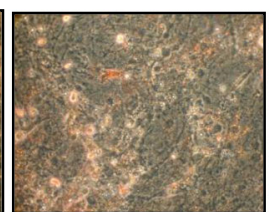

$\mathrm{Fe} 30+\mathrm{D} 20$
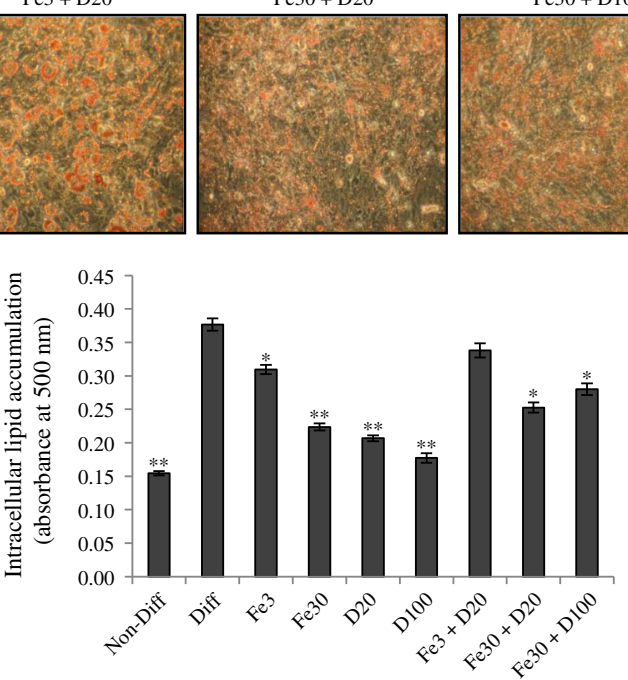

control differentiated; Fe3, FeSO4 (3 $\mu \mathrm{g} / \mathrm{ml})$; Fe30, FeSO4 (30 $\mu \mathrm{g} / \mathrm{ml})$; D20, deferoxamine $(20 \mu \mathrm{mol} / \mathrm{l})$; D100, deferoxamine $(100 \mu \mathrm{mol} / \mathrm{l})$. ${ }^{*} p<0.05, * * p<0.01$ compared with Diff. These data are expressed as mean \pm SEM

and $L e p$ ) gene expression. In fact, both $T f$ gene expression and the release of transferrin protein increased during adipocyte differentiation in parallel with adipogenic genes (in both 3T3L1 cells and human adipocytes). Administration of transferrin, as an iron donor, is well known to facilitate adipocyte differentiation $[28,29]$, but we found no studies of the possible role of endogenous adipocyte transferrin. In fact, human adipose tissue transferrin gene and protein expression was found to be associated positively with adipogenic, and negatively with inflammatory, gene expression [15]. Interestingly, the circulating transferrin concentration correlated significantly with SAT $T F$ gene expression in obese individuals [15], suggesting a strong relationship between circulating and adipose tissue transferrin. Establishing a parallelism with transferrin, the gene expression of another iron donor, lactoferrin (a homologue protein), increased during adipocyte differentiation [25], its administration led to enhanced human adipocyte differentiation [30] and its KD also impaired adipocyte differentiation [31]. 

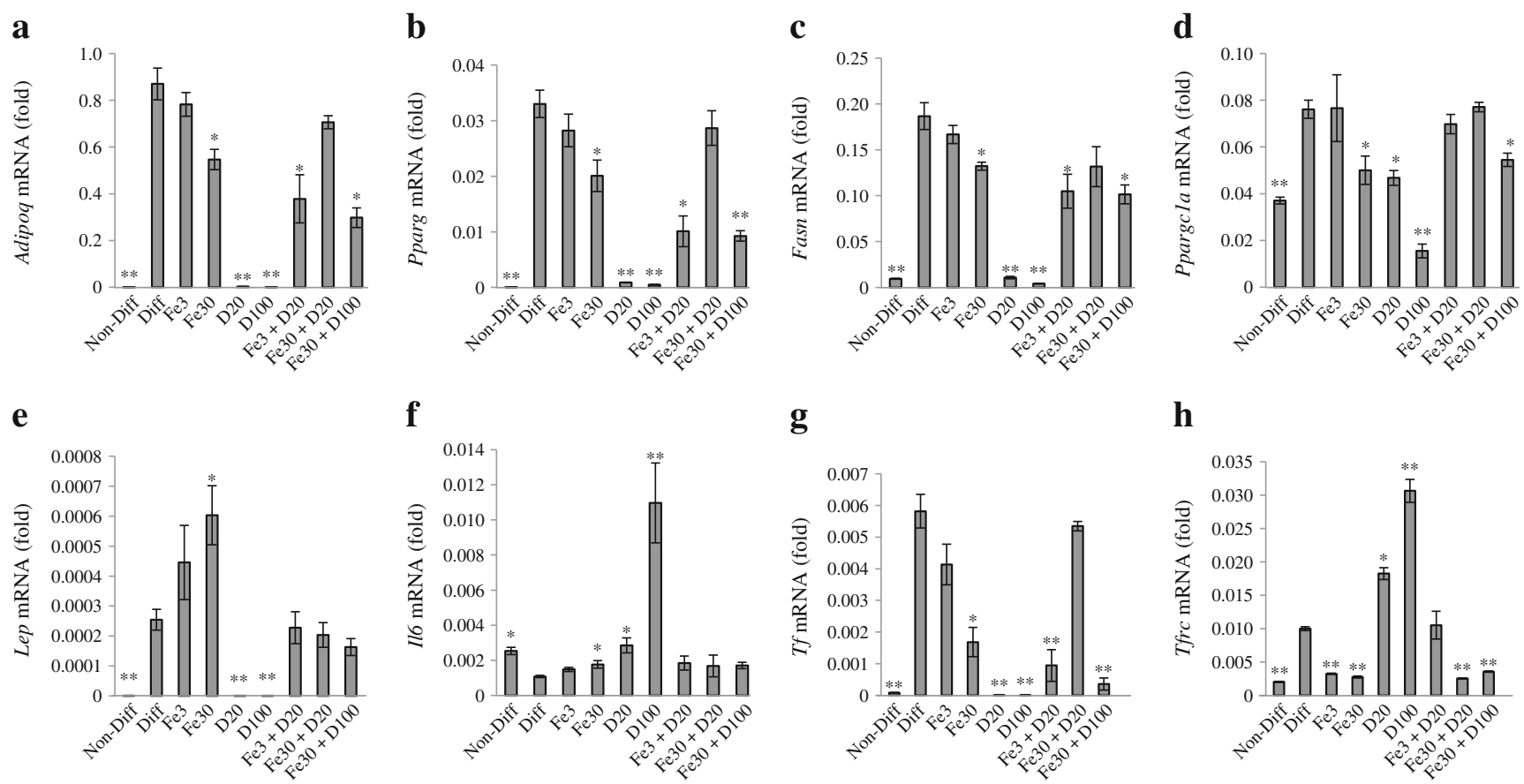

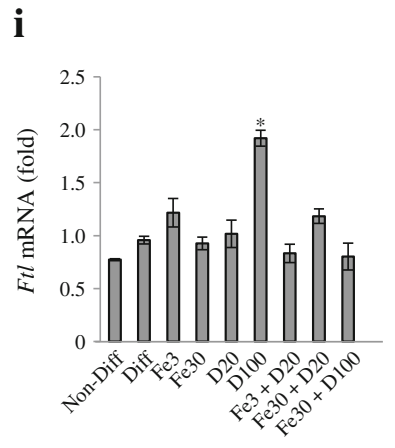

j

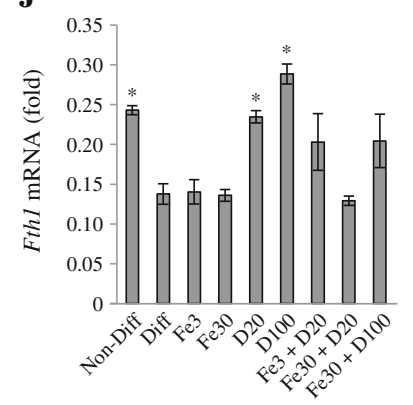

$\mathbf{k}$

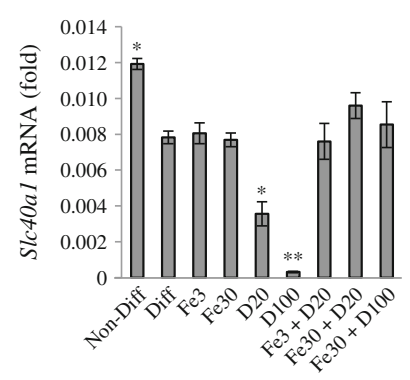

Fig. 5 Effects of FeSO4 (3 and $30 \mu \mathrm{g} / \mathrm{ml}$ ) and deferoxamine (20 and $100 \mu \mathrm{mol} / \mathrm{l}$ ) on Adipoq (a), Pparg (b), Fasn (c), Ppargcla (d), Lep (e), Il6 (f) $T f(\mathbf{g}), T f r c(\mathbf{h}), F t l(\mathbf{i}), F t h l(\mathbf{j})$ and Slc40al (k) gene expression during 3T3-L1 pre-adipocyte differentiation at day 7. Non-Diff, control nondifferentiated; Diff, control differentiated; Fe3, FeSO4 (3 $\mu \mathrm{g} / \mathrm{ml})$; Fe30,

Intriguingly, FTL KD did not significantly affect adipocyte differentiation (present report).

On the other hand, iron chelation resulted in increased TFRC (an iron importer) and decreased SLC40A1 (iron exporter), as expected, according to intracellular iron requirements. Strikingly, iron chelation led to increased FTH1 and FTL mRNA levels in a proportion similar to other inflammatory mediators while decreasing $T F$, in parallel with adipogenic genes and intracellular lipid accumulation. Iron chelation is known to markedly increase phosphorylation and activation of proinflammatory kinases, such as stressactivated protein kinases, JNK and p38MAPK, in several cell lines [32]. In human adipose tissue, FTH1 and FTL gene expression were positively associated with expression of inflammatory genes [15], suggesting that FTH and FTL in adipocytes might be modulated by inflammatory pathways
FeSO4 (30 $\mu \mathrm{g} / \mathrm{ml})$; D20, deferoxamine $(20 \mu \mathrm{mol} / \mathrm{l})$; D100, deferoxamine $(100 \mu \mathrm{mol} / \mathrm{l}) .{ }^{*} p<0.05, * * p<0.01$ compared with Diff. These data are expressed as mean $\pm \mathrm{SEM}$. Relative gene expression is expressed as fold change (fold)

and not merely by the classical regulator of intracellular iron metabolism (iron-related protein 1) [33].

A different gene expression pattern for FTL and SLC40A1 was found during adipocyte differentiation of human and 3T3L1 cells. In human pre-adipocytes, FTL and SLC40A1 gene expression increased in the first stage of adipocyte differentiation and tended to decrease thereafter, whereas it followed the opposite pattern in 3T3-L1 pre-adipocytes. This finding suggests increased iron requirements for 3T3-L1 adipocyte differentiation compared with human pre-adipocytes. Interestingly, in both TF and FTL KD cells, the highest dose of iron was required to increase the expression of adipogenic genes, in marked contrast with control 3T3-L1 cells, in which differentiation was impaired. These findings reveal that the iron requirements for adipocyte differentiation were in fact increased in TF and FTL KD cells. 

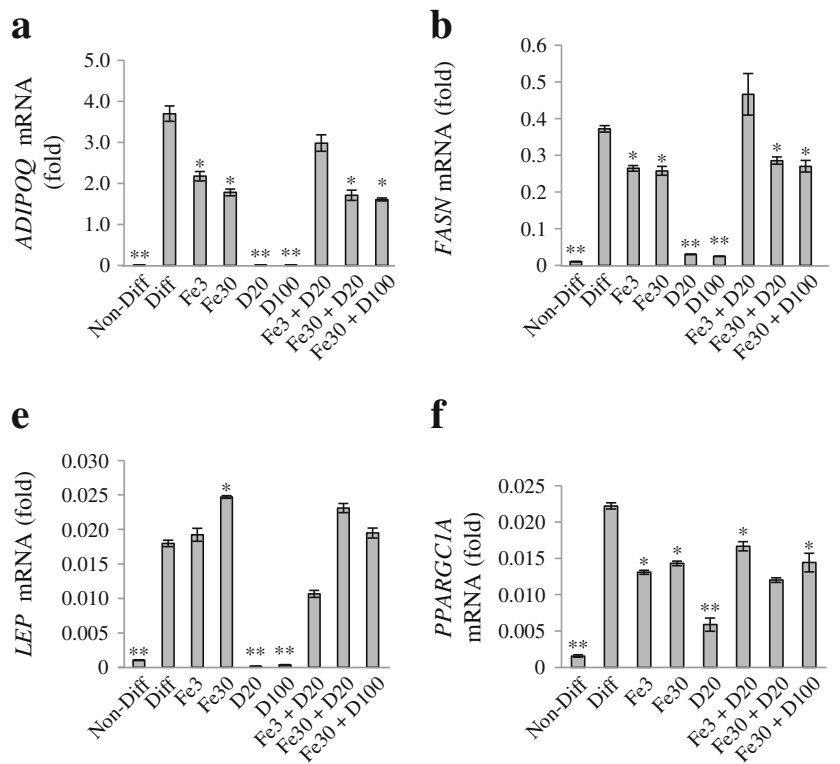

f
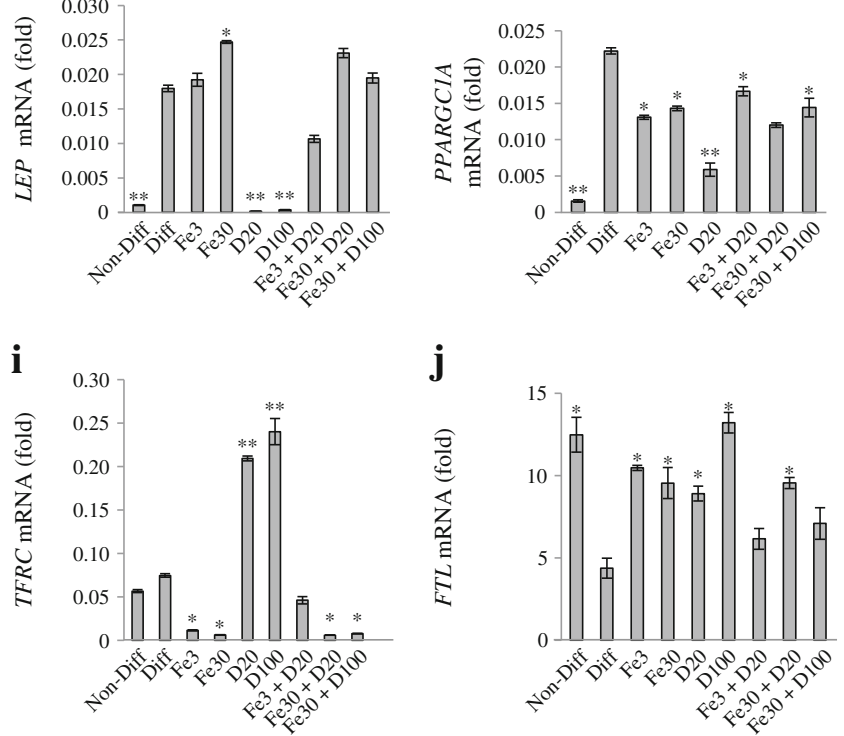

Fig. 6 Effects of FeSO4 (3 and $30 \mu \mathrm{g} / \mathrm{ml}$ ) and deferoxamine (20 and $100 \mu \mathrm{mol} / \mathrm{l}$ ) on ADIPOQ (a), FASN (b), GLUT4 (c), PPARG (d), LEP (e), PPARGC1A (f), IL6 (g), TF (h), TFRC (i), FTL (j), FTH1 (k) and SLC40A1 (I) gene expression during human subcutaneous pre-adipocyte differentiation at day 14. Non-Diff, control non-differentiated; Diff,

Taken together, our present and previous results [15] suggest that obesity might reduce intracellular iron availability, leading to increased expression of inflammatory genes or vice versa. Supporting this hypothesis, palmitate administration led to decreased $T f$ gene expression and iron uptake during adipocyte differentiation. These results are, to some extent, surprising. Endogenous transferrin production and secretion by the adipocytes may result in subsequent transferrin-iron uptake from the medium in normal cells. This uptake would be decreased when the production of endogenous transferrin is limited in TF KD cells, resulting in a smaller uptake in a medium in which no transferrin is produced. Translation of these effects to whole body physiology should be studied in more depth. It is possible that relative extracellular transferrin deficiency in adipose tissue of metabolically compromised individuals influences adipocyte differentiation. Of note, the maximum dose of palmitate displayed similar effects to deferoxamine administration, leading to an iron deficiency phenotype (increased Tfrc and decreased Slc40al mRNA and intracellular iron levels) in parallel with increased $I l 6$, which c

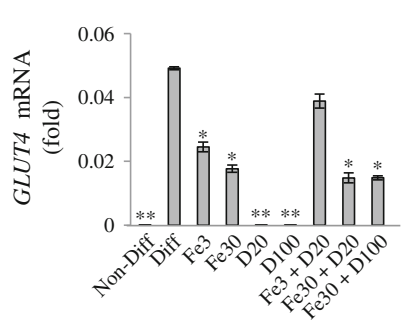

g

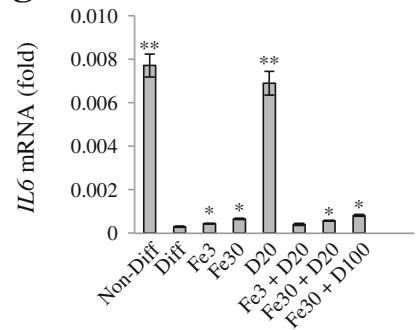

$\mathbf{k}$

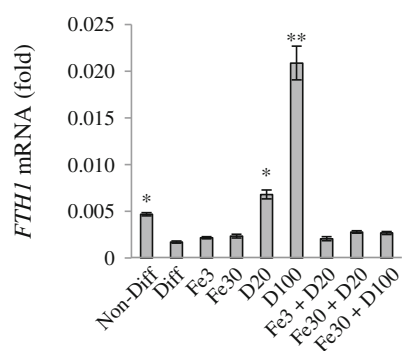

d

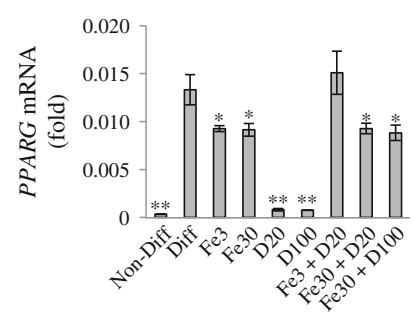

h

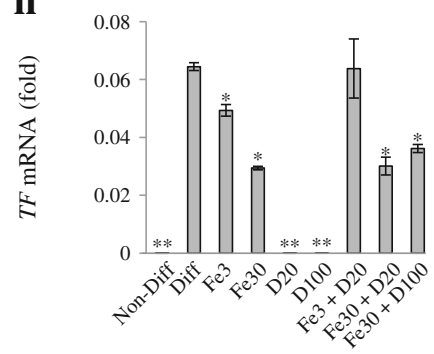

l

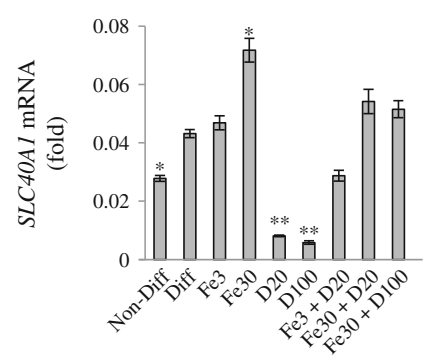

control differentiated; Fe3, FeSO4 (3 $\mu \mathrm{g} / \mathrm{ml})$; Fe30, FeSO4 (30 $\mu \mathrm{g} / \mathrm{ml})$; D20, deferoxamine $(20 \mu \mathrm{mol} / \mathrm{l})$; D100, deferoxamine $(100 \mu \mathrm{mol} / \mathrm{l})$. ${ }^{*} p<0.05,{ }^{*} p<0.01$ compared with Diff. These data are expressed as mean \pm SEM. Relative gene expression is expressed as fold change (fold)

were reversed with holo-transferrin co-administration. In agreement with the present data, obesity in rats induced by a high-fat diet led to increased iron requirements in association with greater accretion of body mass and vulnerability to iron deficiency [34]. The negative effects of palmitate in adipogenesis are mediated in part by the induction of inflammatory pathways and concomitant insulin resistance [35, 36]. Moreover, we found that intracellular iron deficiency induced gene expression of inflammatory cytokines, leading us to suggest that iron deficiency might exacerbate the proinflammatory effects of saturated fatty acids. However, additional in vivo studies are needed to explore the effects of high-fat diet and obesity on adipocyte and adipose tissue iron homeostasis.

Iron in excess also blunts adipogenesis Iron in excess also led to impaired differentiation of both human and 3T3-L1 cells. However, this antiadipogenic effect was less pronounced than under iron chelation. Iron overload in mice is known to decrease adiponectin gene expression in association with insulin resistance [13] and to lead to weight gain, adipocyte 

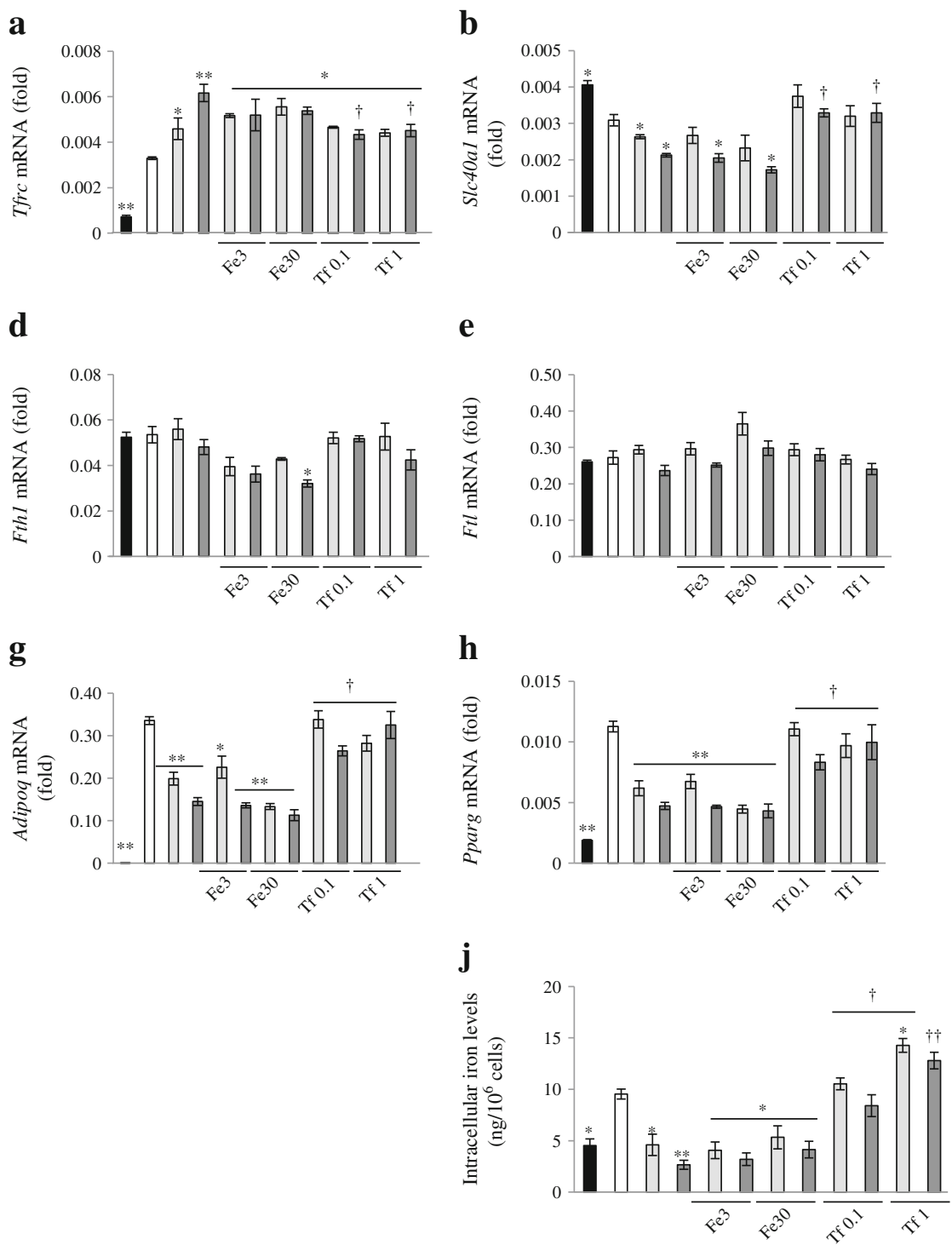

c

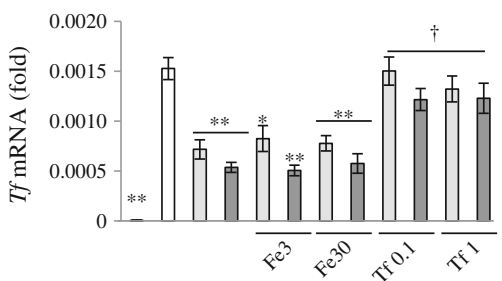

f

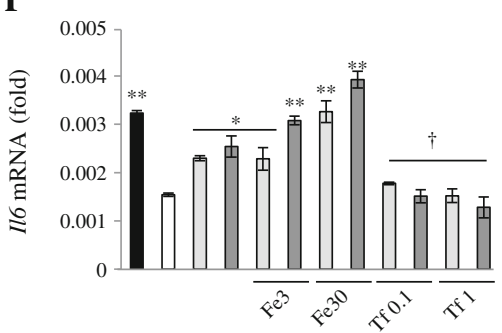

i

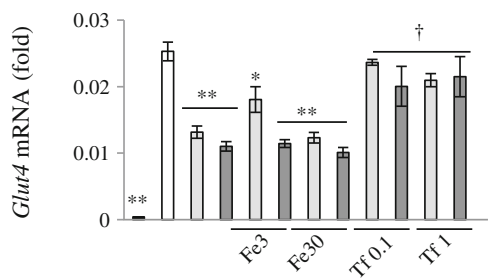

Fig. 7 Effects of palmitate administration (100 and $250 \mu \mathrm{mol} / \mathrm{l})$ and $\mathrm{FeSO}_{4}(3$ and $30 \mu \mathrm{g} / \mathrm{ml} ; \mathrm{Fe} 3$ and $\mathrm{Fe} 30)$ and holo-transferrin $(0.1$ and $1 \mu \mathrm{g} / \mathrm{ml}$; Tf0.1 and Tfl) co-administration on Tfrc (a), Slc40al (b), Tf(c), Fth1 (d), Ftl (e), Il6 (f), Adipoq (g), Pparg (h) and Glut4 (i) gene expression and on intracellular iron levels (j) during 3T3-L1 pre-adipocyte differentiation at day 7. Control non-differentiated (Non-Diff, black

hypertrophy, adipose tissue macrophage infiltration, oxidative stress and metabolic disturbances [37]. In this last model, iron depletion reversed these phenotypes [38]. Adipocyte hypertrophy, which is the 'diabetogenic' adipocyte phenotype, is characterised by increased leptin and reduced adiponectin biosynthesis [39]. In the present study, we found that iron administration exerted inverse effects on $\angle E P$ and $A D I P O Q$ gene expression. Exogenous iron administration led to an increase in $L E P$ gene expression similar to inflammatory genes, whereas iron also resulted in decreased adiponectin and other adipogenic genes. These results suggest that iron overload induced a diabetogenic adipocyte phenotype. These data are in full agreement with previous studies $[13,37]$. bars), control differentiated (Diff, white bars), palmitate (100 $\mu \mathrm{mol} / 1$, light grey bars) and palmitate $(250 \mu \mathrm{mol} / 1$, dark grey bars $)$. Fe3, FeSO4 $(3 \mu \mathrm{g} / \mathrm{ml}) ; \mathrm{Fe} 30, \mathrm{FeSO} 4(30 \mu \mathrm{g} / \mathrm{ml}) .{ }^{*} p<0.05,{ }^{* *} p<0.01$ compared with Diff. ${ }^{\dagger} p<0.05,{ }^{\dagger} p<0.01$ compared with palmitate administration. These data are expressed as mean \pm SEM. Relative gene expression is expressed as fold change (fold)

Mitochondrial biogenesis: a possible link between iron and adipogenesis The antiadipogenic effect of iron chelation might be caused by disruption of mitochondrial biogenesis. Supporting this hypothesis, findings in human adipose tissue revealed that ISCA2 (an important mediator in Fe-S cluster biogenesis) and $T F$ gene expression were positively associated with expression of PPARGC1A, whereas the expression of CYBA, which is a marker for intracellular iron accumulation and induced by oxidative stress $[15,28]$, was negatively linked. $T F$ gene expression was positively associated with other genes related to mitochondrial activity and the respiratory chain (such as PPARGC1B and MT-CO3). As mentioned above, in parallel with the association between transferrin and 
Table 1 Anthropometric and clinical variables of the human cohort $(n=43)$

\begin{tabular}{|c|c|}
\hline Variable & Mean $\pm \mathrm{D}$ \\
\hline Age (years) & $45.3 \pm 11.2$ \\
\hline BMI $\left(\mathrm{kg} / \mathrm{m}^{2}\right)$ & $43.6 \pm 7.7$ \\
\hline Fat mass $(\%)$ & $54.17 \pm 11.2$ \\
\hline Fasting glucose $(\mathrm{mmol} / \mathrm{l})$ & $5.31 \pm 0.78$ \\
\hline $\mathrm{HbA}_{1 \mathrm{c}}(\%)$ & $4.98 \pm 0.49$ \\
\hline $\mathrm{HbA}_{1 \mathrm{c}}(\mathrm{mmol} / \mathrm{mol})$ & $31 \pm 1.4$ \\
\hline Total cholesterol (mmol/l) & $5.01 \pm 0.85$ \\
\hline HDL-cholesterol (mmol/l) & $1.41 \pm 0.39$ \\
\hline LDL-cholesterol (mmol/l) & $2.99 \pm 0.83$ \\
\hline Fasting triacylglycerol (mmol/l) & $1.25 \pm 0.49$ \\
\hline
\end{tabular}

mitochondrial biogenesis, $T F$ gene expression was also significantly associated with adipose tissue functionality (increased adipogenesis and decreased inflammation) [15]. In 3T3-L1 in vitro experiments, iron chelation and TF and FTL KD cells, which had increased iron requirements compared with control cells, showed decreased expression of Ppargcl $\alpha$ (the master activator of mitochondrial biogenesis [23]) and Tfam (a crucial transcription factor for mitochondrial DNA maintenance and biogenesis [40]) during adipocyte differentiation. In addition, iron chelation decreased expression of mitochondrial-related genes (PPARGC1A, PPARGC1B and MT-CO3) during human adipocyte differentiation. These findings hint at the possibility that iron-associated mitochondrial biogenesis during adipocyte differentiation is a factor linking these processes.

To sum up, all these data emphasise that iron plays an important role in adipogenesis. While iron in excess had detrimental effects on adipocyte differentiation, iron deprivation (TF KD, deferoxamine) prevented this process completely. Precise and fine-tuned iron availability is essential to achieve optimal adipocyte differentiation, possibly modulating adipocyte mitochondrial biogenesis.

Acknowledgements We acknowledge the technical assistance of E. Loshuertos and O. Rovira (both from Endocrinology, IdIBGi, Spain).

Funding This work was partially supported by research grants from the Ministerio de Economía y Competitividad (PI11-00214 and PI12/02631). CIBEROBN Fisiopatología de la Obesidad y Nutrición is an initiative of the Instituto de Salud Carlos III.

Duality of interest The authors declare that there is no duality of interest associated with this manuscript.

Contribution statement JMM-N, WR and JMF-R participated in study conception and design. JMM-N, FO and MM participated in data collection and analysis. JMM-N, FO, MM, WR and JMF-R participated in manuscript preparation, drafting the article or revising it critically for important intellectual content, and in final approval of the version to be published. JMM-N and JMF-R are the guarantors of this work.

\section{References}

1. Salonen JT, Tuomainen T-P, Nyyssönen K, Lakka H-M, Punnonen K (1999) Relation between iron stores and non-insulin-dependent diabetes in men: case-control study. Br Med J 317:727-730

2. Jehn M, Clark JM, Guallar E (2004) Serum ferritin and risk of the metabolic syndrome in U.S. adults. Diabetes Care 27:2422-2428

3. Fernández-Real JM, Ricart W, Arroyo E et al (1998) Serum ferritin as a component of the insulin resistance syndrome. Diabetes Care 21: $62-68$

4. Haap M, Fritsche A, Mensing HJ, Häring HU, Stumvoll M (2003) Association of high serum ferritin concentration with glucose intolerance and insulin resistance in healthy people. Ann Intern Med 139: 869-871

5. Fernández-Real JM, López-Bermejo A, Ricart W (2005) Iron stores, blood donation, and insulin sensitivity and secretion. Clin Chem 5: 11201-11205

6. Fernández-Real JM, López-Bermejo A, Ricart W (2002) Cross-talk between iron metabolism and type 2 diabetes. Diabetes 51:23482354

7. Fernández-Ral JM, Manco M (2014) Iron overload impact on chronic metabolic disease. Lancet Diabetes Endocrinol 2:513-526

8. Dekker LH, Nicolaou M, van der A DL et al (2013) Sex differences in the association between serum ferritin and fasting glucose in type 2 diabetes among South Asian Surinamese, African Surinamese, and Ethnic Dutch: the population-based SUNSET study. Diabetes Care 36:965-971

9. Iwasaki T, Nakajima A, Yoneda M et al (2005) Serum ferritin is associated with visceral fat area and subcutaneous fat area. Diabetes Care 28:2486-2491

10. Wlazlo N, van Greevenbroek MM, Ferreira I et al (2013) Iron metabolism is associated with adipocyte insulin resistance and plasma adiponectin: the Cohort on Diabetes and Atherosclerosis Maastricht (CODAM) study. Diabetes Care 36:309-315

11. Festa M, Ricciardelli G, Mele G, Pietropaolo C, Ruffo A, Colonna A (2000) Overexpression of $\mathrm{H}$ ferritin and up-regulation of iron regulatory protein genes during differentiation of 3T3-L1 pre-adipocytes. J Biol Chem 275:36708-36712

12. Dongiovanni P, Ruscica M, Rametta R et al (2013) Dietary iron overload induces visceral adipose tissue insulin resistance. Am J Pathol 182:2254-2263

13. Gabrielsen JS, Gao Y, Simcox JA et al (2012) Adipocyte iron regulates adiponectin and insulin sensitivity. J Clin Invest 122: $3529-3540$

14. Orr JS, Kennedy A, Anderson-Baucum EK et al (2013) Obesity alters adipose tissue macrophage iron content and tissue iron distribution. Diabetes 63:421-432

15. Moreno-Navarrete JM, Novelle MG, Catalán V et al (2014) Insulin resistance modulates iron-related proteins in adipose tissue. Diabetes Care 37:1092-1100

16. Rensvold JW, Ong SE, Jeevananthan A, Carr SA, Mootha VK, Pagliarini DJ (2013) Complementary RNA and protein profiling identifies iron as a key regulator of mitochondrial biogenesis. Cell Rep 3:237-245

17. Hausmann A, Samans B, Lill R, Mühlenhoff U (2008) Cellular and mitochondrial remodeling upon defects in iron-sulfur protein biogenesis. J Biol Chem 283:8318-8330

18. Medina-Gómez G (2012) Mitochondria and endocrine function of adipose tissue. Best Pract Res Clin Endocrinol Metab 26:791-804

19. Choo HJ, Kim JH, Kwon OB et al (2006) Mitochondria are impaired in the adipocytes of type 2 diabetic mice. Diabetologia 49: 784-791

20. Rong JX, Qiu Y, Hansen MK et al (2007) Adipose mitochondrial biogenesis is suppressed in $\mathrm{db} / \mathrm{db}$ and high-fat diet-fed mice and improved by rosiglitazone. Diabetes 56:1751-1760 
21. Bogacka I, Xie H, Bray GA, Smith SR (2005) Pioglitazone induces mitochondrial biogenesis in human subcutaneous adipose tissue in vivo. Diabetes 54:1392-1399

22. Qatanani M, Tan Y, Dobrin R et al (2013) Inverse regulation of inflammation and mitochondrial function in adipose tissue defines extreme insulin sensitivity in morbidly obese patients. Diabetes 62: 855-863

23. Puigserver P, Wu Z, Park CW, Graves R, Wright M, Spiegelman BM (1998) A cold-inducible coactivator of nuclear receptors linked to adaptive thermogenesis. Cell 92:829-839

24. Xu XJ, Gauthier MS, Hess DT et al (2012) Insulin sensitive and resistant obesity in humans: AMPK activity, oxidative stress, and depot-specific changes in gene expression in adipose tissue. J Lipid Res 53:792-801

25. Moreno-Navarrete JM, Serrano M, Sabater M et al (2013) Study of lactoferrin gene expression in human and mouse adipose tissue, human pre-adipocytes and mouse 3T3-L1 fibroblasts. Association with adipogenic and inflammatory markers. J Nutr Biochem 24: 1266-1275

26. Guo W, Wong S, Xie W, Lei T, Luo Z (2007) Palmitate modulates intracellular signaling, induces endoplasmic reticulum stress, and causes apoptosis in mouse 3T3-L1 and rat primary preadipocytes. Am J Physiol Endocrinol Metab 293: E576-E586

27. Koutsari C, Mundi MS, Ali AH, Patterson BW, Jensen MD (2013) Systemic free fatty acid disposal into very low-density lipoprotein triglycerides. Diabetes 62:2386-2395

28. Tajima S, Ikeda Y, Sawada K et al (2012) Iron reduction by deferoxamine leads to amelioration of adiposity via the regulation of oxidative stress and inflammation in obese and type 2 diabetes KKAy mice. Am J Physiol Endocrinol Metab 302: E77-E86

29. Zhang LH, Zhang LJ, Wang Q, Wang B, Yang GS (2008) Expression of TGH and its role in porcine primary adipocyte lipolysis. Mol Cell Biochem 315:159-167
30. Hemmrich K, von Heimburg D, Cierpka K, Haydarlioglu S, Pallua N (2005) Optimization of the differentiation of human preadipocytes in vitro. Differentiation 73:28-35

31. Moreno-Navarrete JM, Ortega F, Sabater M, Ricart W, Fernández-Real JM (2011) Proadipogenic effects of lactoferrin in human subcutaneous and visceral preadipocytes. J Nutr Biochem 22:1143-1149

32. Moreno-Navarrete JM, Ortega F, Moreno M, Serrano M, Ricart W, Fernández-Real JM (2014) Lactoferrin gene knockdown leads to similar effects to iron chelation in human adipocytes. J Cell Mol Med 18:391-395

33. Yu Y, Richardson DR (2011) Cellular iron depletion stimulates the JNK and $\mathrm{p} 38$ MAPK signaling transduction pathways, dissociation of ASK1thioredoxin, and activation of ASK1. J Biol Chem 286:15413-15427

34. Wang J, Pantopoulos K (2011) Regulation of cellular iron metabolism. Biochem J 434:365-381

35. Bertinato J, Aroche C, Plouffe LJ et al (2014) Diet-induced obese rats have higher iron requirements and are more vulnerable to iron deficiency. Eur J Nutr 53:885-895

36. Nguyen MT, Satoh H, Favelyukis S et al (2005) JNK and tumor necrosis factor-alpha mediate free fatty acid-induced insulin resistance in 3T3-L1 adipocytes. J Biol Chem 280:35361-35371

37. Kennedy A, Martinez K, Chuang CC, LaPoint K, McIntosh M (2009) Saturated fatty acid-mediated inflammation and insulin resistance in adipose tissue: mechanisms of action and implications. J Nutr 139:1-4

38. Tinkov AA, Polyakova VS, Nikonorov AA (2013) Chronic administration of iron and copper potentiates adipogenic effect of high fat diet in Wistar rats. Biometals 26:447-463

39. Finucane FM, Luan J, Wareham NJ et al (2009) Correlation of the leptin:adiponectin ratio with measures of insulin resistance in nondiabetic individuals. Diabetologia 52:2345-2349

40. Larsson NG, Wang J, Wilhelmsson H et al (1998) Mitochondrial transcription factor A is necessary for mtDNA maintenance and embryogenesis in mice. Nat Genet 18:231-236 